\title{
Social interactions and query analysis in an online forum
}

\author{
Oladosu Olakunle Abimbola, Okikiola Folasade Mercy, Alakiri Harrison Osarenren, \\ Oladiboye Olasunkanmi Esther
}

Department of Computer Technology, Yaba College of Technology, Yaba, Lagos

\section{Email address:}

kunledosu@gmail.com(Oladosu O. A.), olakunlerejoicemysoul@yahoo.com(Oladosu O. A.), sade.mercy@yahoo.com(Okikiola F. M.), halakiri@yahoo.com(Alakiri H. O.), estherworld2001@yahoo.com(Oladiboye O. E.)

\section{To cite this article:}

Oladosu Olakunle Abimbola, Okikiola Folasade Mercy, Alakiri Harrison Osarenren, Oladiboye Olasunkanmi Esther. Social Interactions and Query Analysis in an Online Forum. American Journal of Networks and Communications. Vol. 3, No. 1, 2014, pp. 13-16. doi: 10.11648/j.ajnc.20140301.12

\begin{abstract}
Online social networks (forum) have become extremely popular; numerous sites allow users to interact and share content using social links observing that there are some limitations on most of the social interaction networks. A recent study has demonstrated that the "strength of ties" varies widely, ranging from pairs of users who are best friends to pairs of users who even wished they weren't friends. In order to distinguish between these strong and weak links, researchers have suggested examining the activity networks, the network that is formed by users who actually interact using one or many of the methods provided by the social networking site. Also for effective study, we collect data on both friendship relationship and interactions for a large subset of the Facebook social network and forum, Twitter, 2go, m.eskimi and other social interactive networks. From a technological standpoint, forums or boards are web applications managing user-generated content. Early Internet forums could be described as a web version of an electronic mailing list or newsgroup (such as exist on Usenet); allowing people to post messages and comment on other messages. It has been discovered that Chat forum is one of the newest way in which organization and the business world use now to reach out to their numerous customers in order to get feedback and responses concerning its entire product lines to share of their opinion.
\end{abstract}

Keywords: Content, Blog, Bookmark, Video Conference, Forum, Social Network, Chat

\section{Introduction}

A discussion forum is hierarchical or tree-like in structure: a forum can contain a number of sub forums, each of which may have several topics. Within a forum's topic, each new discussion started is called a thread, and can be replied to by as many people as so wish. Depending on the forum's settings, users can be anonymous or have to register with the forum and then consequently $\log$ in (i.e. in order to post messages). On most forums, users do not have to $\log$ in to read existing messages. Social networks have become a popular way for users to connect, express themselves, and share content. Popular social networks have hundreds of millions of registered users and are growing at a rapid pace. As these networks grow and mature, users have been observed to form hundreds to even thousands of friendship links. For example, a user in Orkut has on average of over 100 friends [23] and the average number of friends in Facebook is over 120. Certain individuals have much higher degrees than the average; in fact, in Flickr we found multiple users who have more than 25,000 friends! While most social networking sites allow only a binary state of friendship, it has been unsurprisingly observed that not all links are created equal. A recent study [14] has demonstrated that the "strength of ties" varies widely, ranging from pairs of users who are best friends to pairs of users who even wished they weren't friends. In order to distinguish between these strong and weak links, researchers have suggested examining the activity networks, the network that is formed by users who actually interact using one or many of the methods provided by the social networking site. While the initial studies on activity networks [24] have brought great insights into how an activity network is structurally different from the social network and how system designers can utilize such information, little attention has been paid to a rather natural and important aspect of user interaction: the fact that the level of interaction between two individuals can vary over time. Also for effective study, we collect data on both 
friendship relationship and interactions for a large subset of the Facebook social network and forum, Twitter, 2go, m.eskimi and other social interactive networks. . In total, we examine over 60,000 users and over 800,000 logged interactions between those users over a period of months. This data set has been made available to the research community in an anonymized form. We make a number of interesting observations.

\section{Online Forum and Social Network Interaction}

The modern forums originated from bulletin boards, and are a technological evolution of the dialup bulletin board system [25]. From a technological standpoint, forums or boards are web applications managing user-generated content [16]. Early Internet forums could be described as a web version of an electronic mailing list or newsgroup (such as exist on Usenet); allowing people to post messages and comment on other messages. Later developments emulated the different newsgroups or individual lists, providing more than one forum, dedicated to a particular topic [14]. Internet forums are prevalent in several developed countries. Japan posts the most with over two million per day on their largest forum called "2channel". China also has many millions of posts on forums such as "Tianya Club". Forums perform a function similar to that of dial-up bulletin board systems and Usenet networks that were first created starting in the late 1970s [13].

Early web-based forums date back as far as 1994, with the WIT [26] project from W3 Consortium and starting from this time, many alternatives were created [9]. A sense of virtual community often develops around forums that have regular users. Technology, video games, sports, music, fashion, religion, and politics are popular areas for forum themes, but there are forums for a huge number of topics. Internet slang and image macros popular across the Internet are abundant and widely used in Internet forums.

\section{Forum Structure}

A forum consists of a tree like directory structure. The top end is "Categories". A forum can be divided into categories for the relevant discussions. Under the categories are sub forums and these sub forums can further have more sub forums. The topics (commonly called threads) come under the lowest level of sub-forums and these are the places under which members can start their discussions or posts. Logically forums are organized into a finite set of generic topics (usually with one main topic) driven and updated by a group known as members, and governed by a group known as moderators. It can also have a graph structure [3]. All message boards will use one of three possible display formats. Each of the three basic message board display formats is;

a. Non-Threaded

\section{b. Semi-Threaded}

c. Fully Threaded

These formats or threads have their own advantages and disadvantages. If messages are not related to one another at all a Non-Threaded format is best. If you have a message topic and multiple replies to that message topic a semithreaded format is best. If you have a message topic and replies to that message topic, and replies to replies, then a fully threaded format is best [18].

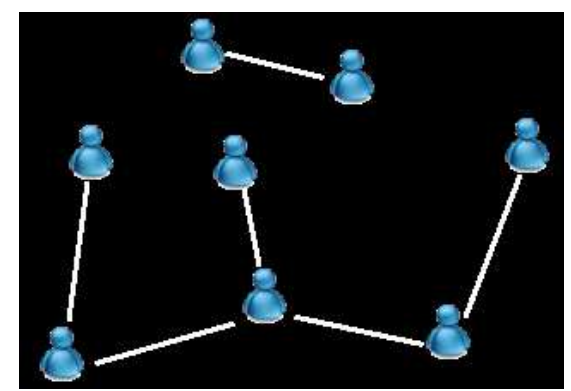

Fig 3.1. Social network

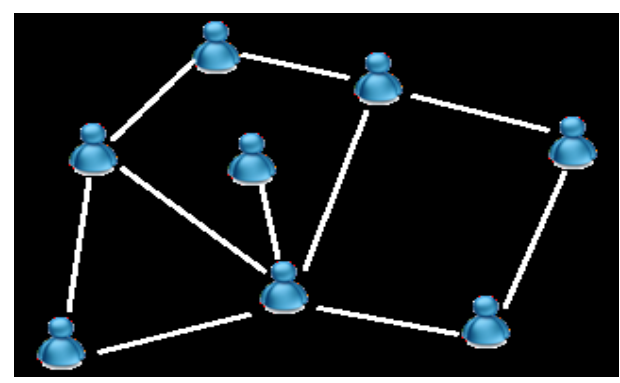

Fig 3.2. Interaction network

\section{Email, IM, Discussion Forums, and Chat}

a. Chat: The term 'chat' refers to an interactive discussion in which all participants are online at the same time, similar to a telephone conversation. Instead of talking into a receiver, however, the chat participants communicate using their keyboards to type comments. Chat is completely interactive, i.e., "live". You can ask questions and make comments and other participants in the chat "room" will be able to see them and reply immediately. Our Women's History Month chats will take place at predetermined times and once they are over, you will not be able to add additional comments or questions to them.

b. Discussion: The term 'discussion' refers to the ongoing forums, like this one, in which you can post comments and questions which will be saved for others to read and comment on in turn. The discussion forums will be available for as long as the WHM website remains online.

c. Email: Is the acronym for "electronic mail" (email is an official English word that requires no hyphen). 
Email is like an old fashioned letter but in electronic format sent from one computer to another. There is no going to the metal mailbox down the road, no envelopes to address and stamps to lick, yet email very much resembles the classic post office mail process. Most importantly: the email recipient does not have to be at their computer for an email to successfully send. Recipients retrieve their email on their own time. Because of this lag between sending and receiving, email is called "non-real time" or "asynchronous time" messaging.

d. Instant Messaging: Unlike email, instant messaging is a real-time messaging format. IM is really a specialized form of 'chat' between people who know each other. Both IM users must be online at the same time for IM to fully work. IM is not as popular as email, but it is popular amongst teenagers and people in office places that allow instant messaging.

e. Discussion Forum: Discussion forums are really a slow-motion form of chat. Forums are designed to build online communities of people with similar interests. Also known as a "discussion group", "board" or "newsgroup", a forum is an asynchronous service where you can trade noninstant messages with other members. The other members reply on their own schedule, and do not need to be present while you are sending. Every forum is also dedicated to some specific community or subject, such as travel, gardening, motorcycles, vintage cars, cooking, social issues, music artists, and more. Forums are very popular, and are renowned for being quite addictive because they put you in touch with many similar-minded people.

\section{Results and Analysis}

Table 5.1. List of queries posted and responses

\begin{tabular}{ccc}
\hline Forum Topic & Number of Posts & $\begin{array}{c}\text { Number of } \\
\text { Responses }\end{array}$ \\
\hline Education & 10 & 30 \\
Technology & 8 & 24 \\
Fashion & 12 & 36 \\
Electronic Gadget & 7 & 21 \\
Automobile & 5 & 15 \\
Shares & 6 & 18 \\
Marriage & 11 & 33 \\
\hline
\end{tabular}

The graph below shows the responses of posts against queries in the chart above. Number of posts is at the Y-axis while number of queries is at the $\mathrm{X}$-axis. They are drawn in ascending order of posts and queries. The graph simply shows that there is tremendous rate at which people respond to topics like Fashion, Marriage, Education, e.t.c. against topic such as Automobile, Shares, e.t.c. based on professionalism, interest and usability of the available topics.

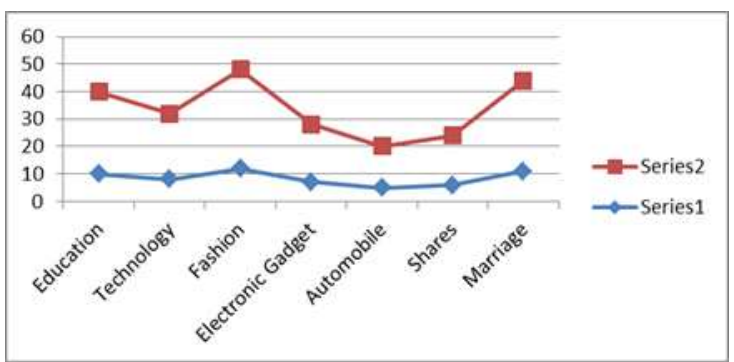

Fig 5.1. Line graph of Queries and Responses

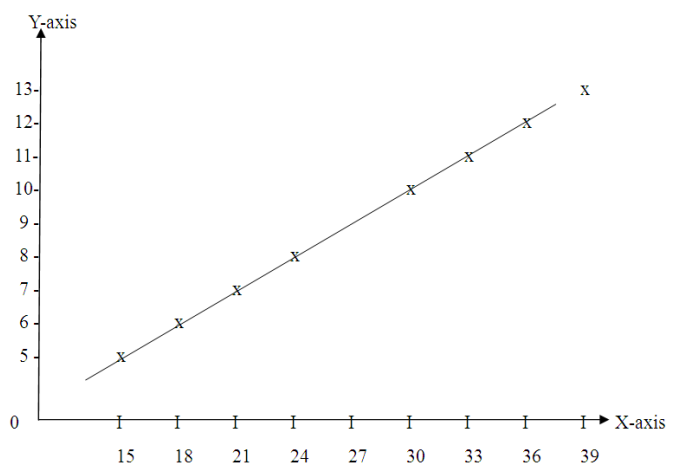

Fig 5.2. Graph of query posts against responses

\section{Interpretation}

Both graphs actually shows that there is a relationship between the number of response received and the query posted i.e. the relative response time to any query post depends on the how interesting is the subject of discourse. Hence, the rate at which users of social network and forum respond to query post is dependent on user's interest in the subject.

\section{Summary and Conclusion}

The study has been all about finding an easier, safer and educative ways of sharing opinions and interact with other people which is commonly refers to as social network (forum). In addition to chatting, this is a new and dynamics means of talking to different people all over the world within a slip of time. It has been discovered that Chat forum is one of the newest way in which organization and the business world use now to reach out to their numerous customers in order to get feedback and responses concerning the entire product lines to share of their opinion.

\section{References}

[1] UITS - Instant Messaging Chatiquette, 2012 - University of Arkansas". Uits.uark.edu. Retrieved 2012-01-19.

[2] IRC Chatiquette - Chat Etiquette, 2012. Livinginternet.com. 1995-11-28. Retrieved 2012-01-19 
[3] LessWrong, 2011 Debate tools: http://wiki.lesswrong.com/wiki/Debate_tools\#ExploreIdeas.com. Retrieved 2011-03-31

[4] http://www.chelseafcforums.com, Retrieved 2011.

[5] Hurrchan/Courtney Wade, 2010 Tripcode Explorer - Hurr Network". $\quad$ http://www.hurrchan.net/wiki/Tripcodes. Retrieved 2010-08-22.

[6] Michael Herman, 2010 "Chat room user guilty of web rage". The Times (London). Retrieved 2010-05-20.

[7] Administrator seeking moderators to control spam abuse, 2010 . ddforums.com. http://www.ddforums.com/showpost.php?p=12566\&postcou $\mathrm{nt}=9$. Retrieved 2010-04-20.

[8] Non-Threaded vs Semi-Threaded vs Threaded Formats, 2010 - BulletinBoards.com". BulletinBoards.com. http://www.bulletinboards.com/ThreadHelp.cfm. Retrieved 2010-05-27.

[9] Forum Software Timeline, 2010. Forum Software Reviews. http://www.forum-software.org/forum-software-timelinefrom-1994-to-today. Retrieved 2010-12-24.

[10] TechnoFyed.com, 2009: Postcount Information: http://www.technofyed.com/showthread.php?tid=219. Retrieved 2009-11-11.

[11] Message Board Features - Website Toolbox, 2009. Website Toolbox.

http://www.websitetoolbox.com/message_board/features.ht ml. Retrieved 2009-07-12

[12] Categories of moderators, 2009: moderators can be global moderators who have rights for the complete forum and only specific to a sub-forum.

[13] Bulletin Community Forum, 2008 - FAQ: What is a bulletin board?".

vBulletin.com.

http://www.vbulletin.com/forum/faq.php?faq=vb3 board_us age\#faq vb3 forums threads posts Retrieved 2008-09-01. "A bulletin board is an online discussion site. It is sometimes also called a 'board' or 'forum'. It may contain several categories, consisting of sub-forums, threads and individual posts."
[14] Gilbert E., Karahalios, Ethan Feerst and Dylan Stewart group, 2008. What is an "Internet forum and video entry?" http://www.videojug.com/expertanswer/internetcommunities-and-forums-2/what-is-an-internet-forum. Retrieved 2008-11-04.

[15] L. Backstrom, R. Kumar, C. Marlow, J. Novak, and A. Tomkins, 2008. Preferential behavior in online groups. In WSDM,

[16] http://bugclub.org/glossary.html. Retrieved 2008

[17] D. Crandall, D. Cosley, D. Huttenlocher, J. Kleinberg, and S Suri, 2008 Feedback e_ects between similarity and social inuence in online communities. In SIGKDD.

[18] Bulletin FAQ: Moderators and Administrators, 2008. vBulletin.com. http://www.vbulletin.com/forum/faq.php?faq=vb3 reading posting\#faq_vb3_mods_admins. Retrieved 2008-09-14.

[19] phpBB FAQ: What is COPPA?". phpBB.com. http://www.phpbb.com/community/faq.php\#f07. Retrieved 2008-09-14

[20] L. Backstrom, D. Huttenlocher, J. Kleinberg, and X. Lan.2006 Group formation in large social networks: membership, growth, and evolution. In SIGKDD, 2006.

[21] Merchant, Guy, 2001. "Teenagers in cyberspace: an investigation of language use and language change in internet chatrooms." Journal of Research in Reading. 2001 Volume 24, Issue 3. ISSN 0141-0423

[22] WIT - WWW Interactive Talk, 1995. W3 Consortium. http://www.w3.org/WIT/. Retrieved 1995-05. http://www.fanforum.com/faq.php?faq=vb_user_maintain\#f aq_vb_why_register

[23] Mislove A. et al, 2007.

[24] Chun H. et al, 2008 and Wilson C. et al 2009.

[25] http://www.videojug.com/expertanswer/internetcommunities-and-forums, Retrieved

[26] http://www.w3.org/WIT/, Retrieved 1995. 